\title{
Mucosal inflammation in Candida esophagitis has distinctive features that may be helpful diagnostically
}

\author{
Isabella W. Martin ${ }^{1} \cdot$ Aaron E. Atkinson $^{1} \cdot$ Xiaoying Liu ${ }^{1}$ Arief A. Suriawinata ${ }^{1}$ Joel A. Lefferts $\mathbb{C}^{1} \cdot$ Mikhail Lisovsky $^{1}$
}

Received: 6 January 2018 / Revised: 28 February 2018 / Accepted: 24 March 2018 / Published online: 19 June 2018

(c) United States \& Canadian Academy of Pathology 2018

\begin{abstract}
The diagnosis of Candida esophagitis can be challenging when the epithelium containing Candida filamentous forms is not readily seen or is entirely sloughed away. Mucosal inflammation could be helpful diagnostically, if distinctive. However it is thought to be nonspecific in Candida esophagitis. The goal of this retrospective study was to identify features of mucosal inflammation helpful in alerting a pathologist to the possibility of Candida esophagitis when Candida mycelia are not readily observed. The study group consisted of 99 consecutive cases of Candida esophagitis and a control group of 64 consecutive cases of reflux esophagitis diagnosed at our institution from 2008-2016. Band-like superficial intraepithelial neutrophils and increased intraepithelial lymphocytes were observed in 75 and $67 \%$ of Candida esophagitis cases, respectively and only in 14 and $19 \%$ of reflux esophagitis cases, respectively $(p<.0001)$. Intraepithelial lymphocytes were peripapillary or CD4-predominant in 75\% of Candida esophagitis cases with increased lymphocytes, in contrast to $17 \%$ of reflux esophagitis cases $(p=.0011)$. Concurrent presence of intraepithelial neutrophils and increased lymphocytes showed increased specificity for Candida esophagitis and was observed in $61 \%$ of patients with Candida esophagitis and only in $2 \%$ of patients with reflux esophagitis $(p<.0001)$. In addition, superficial band-like neutrophils were observed concurrently with increased peripapillary lymphocytes or CD4-predominant lymphocytes in 35 and 50\% of Candida esophagitis cases, respectively, in contrast to no reflux esophagitis cases. Basal cell hyperplasia and elongation of stromal papillae were frequent in both groups. The data suggest that when Candida microorganisms are not readily observed, concurrent presence of superficial band-like neutrophils and increased lymphocytes may be indicative of Candida etiology of active esophagitis.
\end{abstract}

\section{Introduction}

Candida albicans (C. albicans) is a common commensal organism in the oropharyngeal cavity and gastrointestinal tract and is the most common cause of infectious esophagitis [1, 2]. It grows as oval-shaped budding yeast, or as pseudohyphae or true septate hyphae. Both yeast and filamentous forms are usually seen in Candida esophagitis, but only epithelium-invasive filamentous forms are diagnostic of infection, as yeast may superficially colonize the mucosa without causing clinical disease. Although Candida esophagitis may occur in normal individuals, it is more often an opportunistic infection. The development of pathogenicity is enabled by defects in host cellular

Mikhail Lisovsky

Mikhail.lisovsky@hitchcock.org

1 Department of Pathology, Dartmouth-Hitchcock Medical Center and Geisel School of Medicine at Dartmouth, Lebanon, NH, USA immunity, such as immunodeficiency states including AIDS, steroid use, malignancy, poorly-controlled diabetes, antibiotic use and mucosal barrier injury, such as gastroesophageal reflux disease [3,4]. Although $C$. albicans is the most commonly implicated species, others including $C$. dubleniensis, C. tropicalis, C. parapsilosis and C. glabrata have been also described as causative agents of esophagitis, but at a significantly lower rate [5-7]. Patients with Candida esophagitis mainly complain of odynophagia and dysphagia and demonstrate whitish plaques and exudate endoscopically [1, 3].

Pathologic diagnosis of Candida esophagitis is often straightforward: tissue-invasive fungal forms can be readily seen with hematoxylin-eosin stain usually within detached squamous epithelium or debris, which may be admixed with neutrophils and bacteria. Fungal organisms can then be confirmed with special stains like Gomori Methenamine Silver or Periodic Acid Schiff. However, diagnostic epithelium may be scarce and difficult to appreciate, or it may be sloughed away during either endoscopy or biopsy fixation and processing [1]. In these recurring situations, the 
diagnosis may become problematic, with a differential diagnosis including other causes of active esophagitis, notably reflux esophagitis. Mucosal inflammation could be helpful diagnostically, if it had distinctive features. However, mucosal inflammation is thought to be nonspecific in Candida esophagitis, although the supporting data are scarce [2, 8-10]. Surprisingly little is known about histologic features of Candida esophagitis, besides the presence of Candida organisms themselves.

The goal of this study was to identify characteristic features of mucosal inflammation in Candida esophagitis, apart from the fungal microorganisms, in order to aid in differentiating Candida esophagitis from other types of active esophagitis in problematic cases.

\section{Material and methods}

\section{Case selection}

This was an observational retrospective study of 99 consecutive cases of Candida esophagitis identified through searching the laboratory information system from 2008-2014 and during routine diagnostic work from 2015-2016 in a tertiary care academic center. Inclusion criteria consisted of (a) unequivocal presence of Candida filamentous forms in the epithelium; and (b) sufficient squamous mucosa in the biopsy. A control group consisted of 64 consecutive biopsy cases of histologic reflux esophagitis also selected through searching the laboratory information system. The study was approved by the Committee for the Protection of Human Subjects at Dartmouth College.

\section{Clinical information}

Clinical data were extracted from the electronic medical records of Dartmouth-Hitchcock Medical Center and included: age, sex, past medical history, results of standard manometry or barium esophagram, and use of any of the following medications during the 8 weeks preceding biopsy: corticosteroids, antibiotics, immunosuppressive drugs, chemotherapeutic drugs and biologically active agents. To ensure proper recording of retrospective data, a uniform electronic data abstraction form with data codes and a protocol to resolve ambiguous or conflicting data was created. The abstractors (IM and ML) were blinded to the clinical and histologic data.

\section{Histologic evaluation}

Candida esophagitis was defined as the presence of pseudohyphae consistent with Candida species invading the esophageal squamous epithelium. Diagnostic features of reflux esophagitis included basal hyperplasia (expansion of the basal zone more than $15 \%$ of the thickness of the squamous epithelium), elongated stromal papillae (lengthening of the stromal papillae to more than two thirds of the thickness of squamous epithelium) and/or intraepithelial granulocytes [11]. The following histologic features were evaluated: basal cell hyperplasia and elongation of stromal papillae, distribution and depth of localization of intraepithelial neutrophils; number of intraepithelial eosinophils and distribution, localization and number of intraepithelial lymphocytes. When no mucosal inflammation was present in the original slide, 3 deeper steps were evaluated.

Intraepithelial neutrophils were categorized as having band-like or focal distribution. Band-like neutrophils continuously involved superficial layers of the epithelium, and the depth of involvement was quantified according to the number of involved epithelial cell layers (parakeratotic layers included, when present). Focal neutrophils showed discrete, discontinuous distribution, not conforming to a band-like appearance. Intraepithelial lymphocytes were counted in the $400 \times$ field of view (high power field) with the highest density of lymphocytes. The upper limit of normal number of intraepithelial lymphocytes was 62 at 0 to $2 \mathrm{~cm}$ (gastroesophageal junction), 46 at $5 \mathrm{~cm}$ (distal esophagus) and 41 at $10 \mathrm{~cm}$ (mid esophagus), as previously reported [12]. The localization of increased lymphocytes was categorized as peripapillary or diffuse. Peripapillary lymphocytes centered on a stromal papilla, with interpapillary area largely devoid of lymphocytes. Diffuse lymphocytes were scattered and had no relation to the papillae. Intraepithelial eosinophils were considered rare when no more than 1 eosinophil on average was observed for every 2 high power fields; more frequent eosinophils were called multiple. Intraepithelial neutrophils and increased intraepithelial lymphocytes were called concurrent when both were present in a biopsy specimen. Intraepithelial neutrophils and increased lymphocytes were called co-localized when they overlapped in the same area of the epithelium.

Biopsies were fixed in $10 \%$ formalin, paraffin-embedded and stained with hematoxylin and eosin. Gomori Methenamine Silver stain was used where appropriate to support the diagnosis of Candida esophagitis. The microscopy was performed by two authors independently (IM and ML) and discrepancies were resolved by consensus. Microscopy was performed using an Olympus BX 41 microscope with a field number 22 eyepiece, resulting in a $0.237 \mathrm{~mm}^{2}$ field of view at $400 \times$ magnification.

\section{Immunohistochemistry}

Routine CD4 and CD8 immunohistochemistry was performed on all cases of Candida esophagitis with increased 
intraepithelial lymphocytes using Bond Polymer Refine Detection staining reagents (Leica Biosystems Newcastle Ltd, Newcastle Upon Tyne, UK) and Bond III autostainer (Leica Microsystems). The following primary antibodies were used: anti-CD4 antibody at a 1:100 dilution (clone SP35, Cell Marque, Rocklin, CA) and anti-CD8 Bond ${ }^{\mathrm{TM}}$ ready-to-use antibody (clone 4b11, Leica Biosystems). CD4 T-cells and CD8 T-cells were counted in the same field of view (400x) where lymphocytes were counted. The CD4: CD8 ratios of $>1$ or $\leq 1$ indicated predominance of CD4 or CD8 T-cells, respectively.

\section{Molecular identification of C. albicans}

DNA from formalin-fixed paraffin-embedded biopsies was extracted as described using QIAamp DNA FFPE tissue kit (Qiagen, Germantown, MD) with the following modifications: proteinase $\mathrm{K}$ incubation extension to $6 \mathrm{~h}$ at $55^{\circ} \mathrm{C}$ followed by addition of 10 units zymolase (Zymo Research, Irvine, CA; Catalog \#E1004,) and $37^{\circ} \mathrm{C}$ overnight incubation prior to extraction [13]. DNA primers were designed to the $C$. albicans-specific gene, EEDl (Epithelial Escape and Dissemination 1): EED1 Forward, AGTGTTGCAAAAGAATACCC, and EED1 Reverse, CATGAGAATTAGTTTGATGAACC [14]. Beta-globin primers were also used for polymerase chain reaction (PCR), as described previously [13]. For PCR, AmpliTaq Gold (Applied Biosystems, Foster City, CA) was used per manufacturer's instructions and primers. PCR products were analyzed using 2\% Egels and Egel sample buffer (Invitrogen, Carlsbad, CA).

\section{Statistics}

The distributions of baseline characteristics were compared between the groups using two sample t-test for continuous variables and Fisher's exact test for categorical variables. Statistical analyses were performed using GraphPad Prism (GraphPad Software Inc., La Jolla, CA)

\section{Results}

\section{General characterization of biopsies with Candida esophagitis}

Of 99 cases of Candida esophagitis, 88 (89\%) had features of mucosal inflammation, such as basal cell hyperplasia, elongated stromal papillae, intraepithelial neutrophils, eosinophils and increased intraepithelial lymphocytes. Eleven cases had no evidence of inflammation even after evaluation of deeper levels. Comparison of cases with extensive involvement by Candida mycelia with those with scarce involvement did not reveal a noticeable difference in the extent of mucosal inflammation.

\section{Histologic features of mucosal inflammation in Candida esophagitis}

The data are presented in Table 1. Basal hyperplasia and elongated stromal papillae were present in $73 \%$ (64/88) and $52 \%$ (46/88) of Candida esophagitis cases, respectively and in $95 \%(61 / 64)$ and $81 \%$ (52/64) of reflux esophagitis cases, respectively $(p<.0007)$. Erosions or evidence of ulcer were not observed in Candida esophagitis cases, but were present in 20\% (13/64) of reflux esophagitis cases $(p<.0001)$. These data suggest that features of epithelial regeneration are common both in Candida esophagitis and reflux esophagitis. However, erosions/ulcers are largely restricted to reflux esophagitis.

Intraepithelial neutrophils were found in 94\% (83/88) of Candida esophagitis cases with mucosal inflammation and only in $22 \%(14 / 64)$ of reflux esophagitis cases $(p<.0001)$. Band-like superficial neutrophils were observed in the majority of Candida esophagitis cases, $75 \%(66 / 88)$, and only in $14 \%(9 / 64)$ of reflux esophagitis cases $(p<.0001$; Fig. 1). In Candida esophagitis, the band width varied from two squamous cell layers to the upper half of the epithelium, with the mean of $3.7 \pm 1.8$ cell layers. This was significantly wider than $2.2 \pm 0.4$ cell layers in reflux esophagitis $(p<.018)$, suggesting that band-like neutrophils are more prevalent and more prominent in Candida esophagitis than in reflux esophagitis.

Candida esophagitis and reflux esophagitis also differed in the content of intraepithelial lymphocytes. The latter were increased (range 50-245 lymphocytes per high power field) in 67\% (59/88) of Candida esophagitis cases and only in $19 \%$ (12/64, range 44-130) of reflux esophagitis cases $(p<.0001)$. Intraepithelial lymphocytes were peripapillary in 75\% (44/59) of Candida esophagitis cases and only $17 \%(11 / 64)$ of reflux esophagitis cases $(p=.0011$; Fig. 2a). In addition, 75\% (44/59) of Candida esophagitis biopsies with increased intraepithelial lymphocytes had predominance of CD4 T-cells $\quad(\mathrm{CD} 4: \mathrm{CD} 8=2.8 \pm 1.7$; Fig. 2b-d), while $83 \%(10 / 12)$ of reflux esophagitis cases had predominance of CD8 T-cells $(\mathrm{CD} 4: \mathrm{CD} 8=0.7 \pm 0.6$, $p=.005)$. In summary, increased intraepithelial lymphocytes were common, mainly peripapillary and CD4predominant in Candida esophagitis, in contrast to reflux esophagitis.

Combination of several features showed increased specificity for Candida esophagitis. Intraepithelial neutrophils and increased lymphocytes were present concurrently in $61 \%$ (54/88) of Candida esophagitis cases, but only in $2 \%$ $(1 / 64)$ of reflux esophagitis cases $(p<.0001$; sensitivity 
Table 1 Histologic features of inflammation in Candida esophagitis and reflux esophagitis
Fig. 1 Mucosal inflammation in Candida esophagitis. a A superficial band-like neutrophilic infiltrate with microabscesses. Elongated stromal papillae and increased lymphocytes are also present. b A superficial band-like neutrophilic infiltrate and basal cell hyperplasia. c An only 1-2 cells-thick band of superficial neutrophils, with basal hyperplasia and increased lymphocytes

\begin{tabular}{|c|c|c|c|}
\hline & $\begin{array}{l}\text { Candida esophagitis, } \% \\
(n=88)\end{array}$ & $\begin{array}{l}\text { Reflux esophagitis, \% } \\
(n=64)\end{array}$ & $P$-value \\
\hline Basal hyperplasia & 73 & 95 & .0007 \\
\hline Elongated papillae & 52 & 81 & .0003 \\
\hline \multicolumn{4}{|l|}{ Intraepithelial neutrophils } \\
\hline Total & 94 & 22 & $<.0001$ \\
\hline Band-like & 75 & 14 & $<.0001$ \\
\hline Patchy/focal & 19 & 8 & .0342 \\
\hline \multicolumn{4}{|l|}{ Increased intraepithelial lymphocytes } \\
\hline Total & $67(59 / 88)$ & $19(12 / 64)$ & $<.0001$ \\
\hline Peripapillary & $75(44 / 59)$ & $17(2 / 12)$ & .0011 \\
\hline Diffuse & $25(14 / 59)$ & $83(10 / 12)$ & .0011 \\
\hline CD4-predominant & $75(44 / 59)$ & $17(2 / 12)$ & .005 \\
\hline $\begin{array}{l}\text { Concurrence of intraepithelial Neutrophils } \\
\text { and increased lymphocytes }\end{array}$ & 61 & 2 & $<.0001$ \\
\hline $\begin{array}{l}\text { Concurrence of band-like neutrophils and } \\
\text { peripapillary lymphocytes }\end{array}$ & 35 & 0 & $<.0001$ \\
\hline $\begin{array}{l}\text { Concurrence of band-like neutrophils and } \\
\text { increased CD4-predominant lymphocytes }\end{array}$ & 50 & 0 & $<.0001$ \\
\hline $\begin{array}{l}\text { Co-localization of intraepithelial neutrophils } \\
\text { and increased lymphocytes }\end{array}$ & 35 & 2 & $<.0001$ \\
\hline \multicolumn{4}{|l|}{ Intraepithelial eosinophils } \\
\hline No & 66 & 25 & $<.0001$ \\
\hline Rare & 20 & 14 & $<.0001$ \\
\hline Multiple & 14 & 39 & $<.0001$ \\
\hline Erosion/ulcer & 0 & 20 & $<.0001$ \\
\hline
\end{tabular}
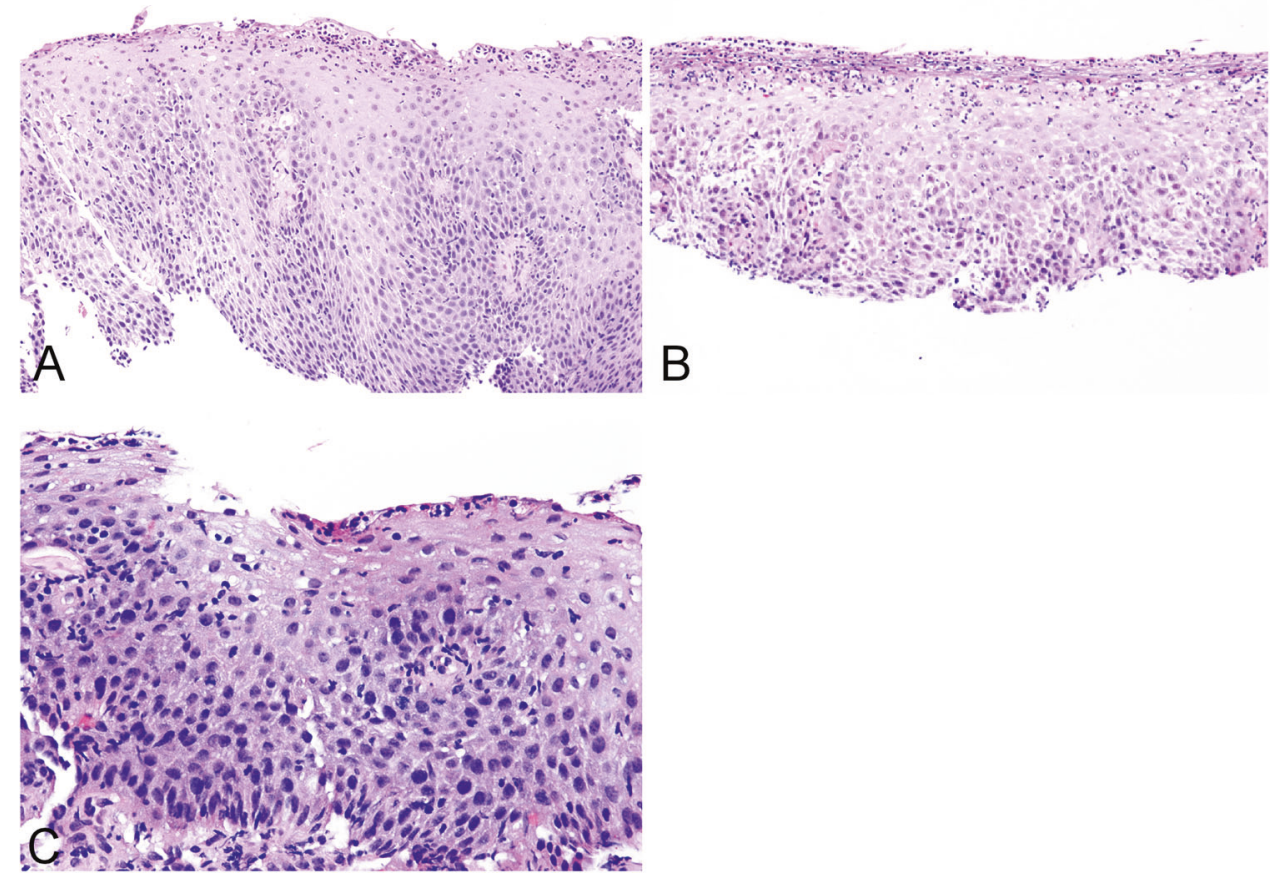
Fig. 2 a, b Increased Co-localization with superficial neutrophils is present in (a). performed on a specimen shown in (b) demonstrates that $\mathrm{CD} 4$ T-cells outnumber CD8 T-cells. c CD4 T-cells. (d) CD8 T-cells peripapillary lymphocytes. c, d Immunohistochemistry
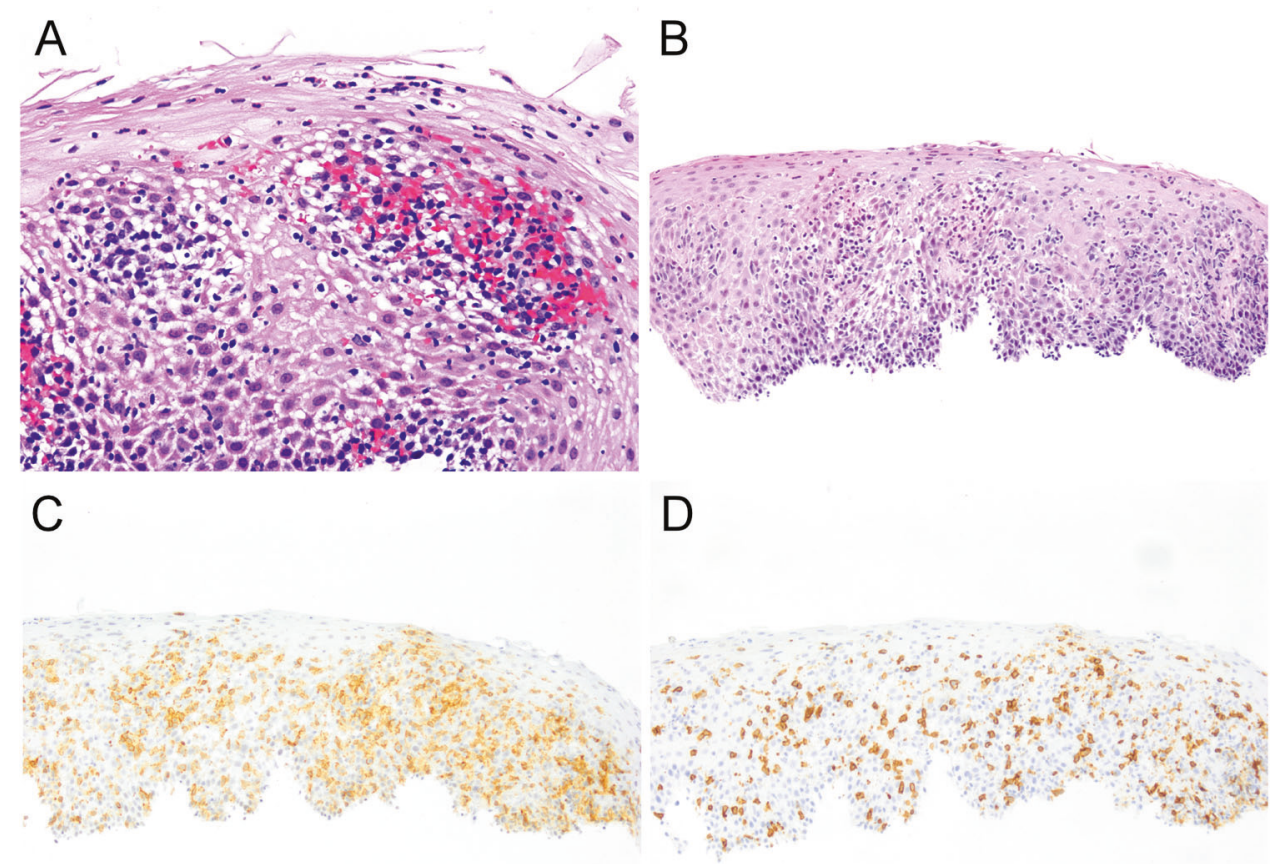

Table 2 Mucosal inflammation in cases with attached to mucosal surface and detached Candida pseudohyphae

\begin{tabular}{lllc}
\hline & Detached Candida pseuhyphae & Attached Candida pseuhyphae & $P$-value \\
\hline Increased lymphocytes & $83 \%(10 / 12)$ & $71 \%(10 / 14)$ & .652 \\
Peripapillary lymphocytes & $70 \%(7 / 10)$ & $60 \%(6 / 10)$ & 1.00 \\
CD4-predominant lymphocytes & $50 \%(5 / 10)$ & $70 \%(7 / 10)$ & .650 \\
Band-like neutrophils & $83 \%(10 / 12)$ & $86 \%(12 / 14)$ & 1.00 \\
Number of cell layers & $3.7 \pm 1.7$ & $3.5 \pm 1.8$ & .709
\end{tabular}

$61 \%$ and specificity 98\%). In addition, concurrence of band-like neutrophils with increased peripapillary lymphocytes or CD4-predominant lymphocytes was observed in 35\% (31/88) and 50\% (44/88) of Candida esophagitis cases, respectively and in 0 of reflux esophagitis cases (sensitivity 35 and 50\%, respectively and specificity $100 \%$ ). These data suggest that Candida-induced inflammation is distinctively characterized by concurrent presence of band-like neutrophils and intraepithelial lymphocytes.

We next wanted to ascertain whether attachment of Candida pseudohyphae to mucosal surface correlated with the degree of mucosal inflammation. Of 26 available cases with Gomori Methenamine Silver stain, 12 cases showed only detached Candida pseudohyphae and 14 cases showed Candida pseudohyphae attached to the surface of mucosal fragments. There was no difference in the degree of inflammation between the groups as assessed by the proportion of cases with increased lymphocytes or band-like neutrophils (Table 2).

\section{Evaluation of motility abnormalities in Candida esophagitis}

Because CD4-predominant peripapillary lymphoid aggregates are characteristic of dysmotility-associated lymphocytic esophagitis [12,15] and similar lymphoid aggregates are observed in Candida esophagitis, we wanted to elucidate whether Candida esophagitis is associated with motility abnormalities. Of 10 patients with Candida esophagitis and mucosal inflammation evaluated by manometry or barium esophagram, $7(70 \%)$ showed various types of motility abnormalities (1-diffuse esophageal spasm, 1 nutcracker esophagus, 1 ineffective motility, 2 motor failure in the body of the esophagus and 2 dysmotility patterns by barium swallow). Six of $10(60 \%)$ evaluated patients with reflux esophagitis also demonstrated various types of motility abnormalities ( 3 ineffective motility, 1 achalasia and 2 dysmotility patterns by barium swallow). These findings do not support an association between Candida esophagitis and esophageal motility abnormalities. 
Table 3 Clinicoepidemiological factors in Candida esophagitis with and without mucosal inflammation

\begin{tabular}{llll}
\hline & $\begin{array}{l}\text { Candida esophagitis with } \\
\text { mucosal inflammation, } \\
(n=88)\end{array}$ & $\begin{array}{l}\text { Candida esophagitis without } \\
\text { mucosal inflammation, } \\
(n=11)\end{array}$ & $\begin{array}{l}P \text {-value } \\
\text { Age (yrs) }\end{array}$ \\
Sex (Male: Female) & $58.3 \pm 17.0$ & $50.4 \pm 22.7$ & .161 \\
Primary immunodeficiency & $42: 52$ & $4: 7$ & .752 \\
Diabetes & $1 \%$ & 0 & 1.000 \\
Malignancy & $23 \%$ & $18 \%$ & 1.000 \\
Alcoholism & $12 \%$ & $18 \%$ & .625 \\
Malnutrition & $8 \%$ & 0 & 1.000 \\
Organ transplant & $3 \%$ & 0 & 1.000 \\
End stage renal disease & $2 \%$ & $10 \%$ & .287 \\
Antibiotics & $2 \%$ & $10 \%$ & .287 \\
Steroids & $16 \%$ & $10 \%$ & 1.000 \\
Chemotherapeutic, & $47 \%$ & $36 \%$ & .541 \\
immunosuppressive and bioactive & $8 \%$ & $18 \%$ & .242 \\
drugs & & & .496 \\
Gastroesophageal reflux disease & $60 \%$ & $55 \%$ & \\
\hline
\end{tabular}

\section{Clinico-epidemiologic and microbiological features of CE with and without mucosal inflammation}

To gain insight into possible causes of mucosal inflammation (or lack thereof) associated with Candida esophagitis, we compared multiple clinico-epidemiologic parameters in 88 patients with mucosal inflammation and 11 patients without mucosal inflammation. Analyzed variables included: age, sex, history of primary immunodeficiency, diabetes, malignancy, alcoholism, malnutrition, organ transplant, end-stage renal disease, gastroesophageal reflux disease, use of antibiotics, steroids, chemotherapeutic drugs, immunosuppressive drugs and bioactive agents. No significant differences were observed between the groups (Table 3).

We then evaluated the possibility that mucosal inflammation is associated with particular Candida species. With that goal, we determined the prevalence of $C$. albicans in 11 cases of Candida esophagitis without inflammation (DNA quality was inadequate in 1 case) and in 13 random cases of Candida esophagitis with inflammation using PCR to detect the $C$. albicans-specific gene, EED1. Similar proportion of DNA samples, $100 \%$ (10/10) and 92\% (12/13), respectively were positive suggesting high prevalence of $C$. albicans in both groups (Fig. 3).

\section{Discussion}

Prior literature has deemed mucosal inflammation of Candida esophagitis to be non-specific. In 1972, Moulinier et al. recognized that occasionally epithelium underlying candidal exudate appeared normal, but more often demonstrated

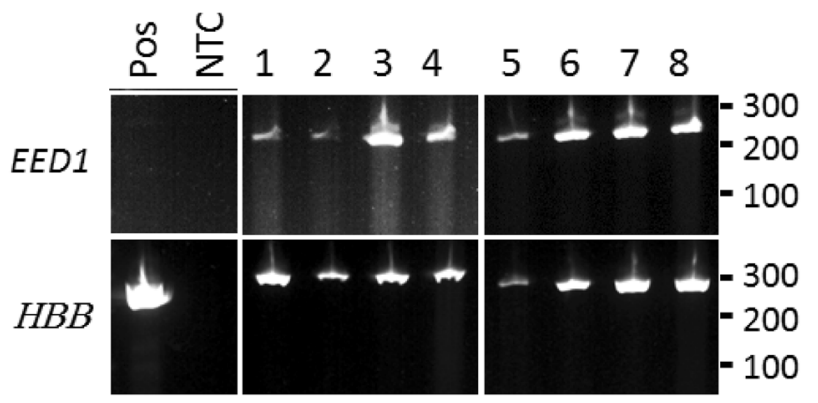

Fig. 3 PCR products (210 base pairs) of C. albicans-specific EED1 gene. Lanes 1-4, samples lacking mucosal inflammation. Lanes 5-8, samples with mucosal inflammation. Also shown are a beta-globin PCR product ( $H B B, 268$ base pairs) used as an internal control, betaglobin positive control (POS), no template control (NTC), and size markers

inflammatory changes with vascular congestion, exudate and mononuclear cell infiltrate [8]. A few years later, Kodsi et al. reported that histology of Candida esophagitis is indistinguishable from reflux esophagitis, except for the presence of Candida [9]. More recent reports describe frequent presence of intraepithelial lymphocytes and neutrophils in Candida esophagitis, but also do not propose any diagnostic value of these findings [2, 10].

Our study confirms similarity of some morphologic features between Candida esophagitis and reflux esophagitis, such as frequent presence of basal hyperplasia and elongated stromal papillae. However, we have also found significant differences. Superficial band-like intraepithelial neutrophils were a characteristic feature of Candida esophagitis in $75 \%$ of patients with mucosal inflammation in contrast to a minority of patients with reflux esophagitis $(14 \%)$. Increased intraepithelial lymphocytes were also very 
common in Candida esophagitis in contrast to reflux esophagitis (67 vs. 19\%). In addition, lymphocytes were mainly peripapillary and CD4-predominant in Candida esophagitis, while they were predominantly diffuse and CD8-predominant in reflux esophagitis. One important distinctive feature of Candida esophagitis that emerged in our analysis is concurrent presence of intraepithelial neutrophils and increased lymphocytes. Even more specific was the concurrence of band-like neutrophils with increased peripapillary or CD4-predominant lymphocytes (35 and $50 \%$, respectively), as it was observed in none of reflux esophagitis cases. Therefore, the concurrent presence of neutrophils and increased lymphocytes should raise the possibility of Candida esophagitis when a diagnosis of reflux esophagitis is contemplated.

In normal subjects, CD4 T-cells are mainly found in the lamina propria and CD8 T-cells in the epithelium $[12,16]$. CD8-predominant intraepithelial lymphocytes are increased in reflux esophagitis and eosinophilic esophagitis and may have a role in the development of these diseases [17-19]. Conversely, the Th17 lineage of CD4 T-lymphocytes plays a key role in the response to mucosal candidiasis by activating neutrophils and antimicrobial factors [20, 21]. Colocalization of CD4-predominant T-lymphocytes with neutrophils in our study is consistent with the importance of CD4 T-cells in the immune response to Candida infection.

Little is known about other types of esophagitis with increased CD4-predominant lymphocytes. An association between a CD4 T-cell-predominant form of lymphocytic esophagitis and primary esophageal motility abnormalities was recently reported $[12,15,22]$. Our data does not support an association between Candida esophagitis and esophageal motility abnormalities, however $3(3.4 \%)$ of our patients with Candida esophagitis had increased peripapillary lymphocytes and no evidence of intraepithelial granulocytes, fulfilling the criteria for lymphocytic esophagitis (data not shown) [23]. In all of these cases the lymphocytes were CD4-predominant. If Candida mycelia had not have been appreciated, the findings could have been interpreted as compatible with dysmotility-associated lymphocytic esophagitis. Thus, Candida esophagitis may join the differential diagnosis of lymphocytic esophagitis. Presence of characteristic endoscopic features of Candida infection (i.e., dome-shaped white plaques) would be crucial for suggesting Candida esophagitis in these cases in the absence of Candida microorganisms. Other associations of lymphocytic esophagitis are with Crohn's disease in pediatric patients and common variable immunodeficiency, however the immunophenotype of lymphocytes in these conditions remains to be fully elucidated $[24,25]$.

Eleven percent of patients with Candida esophagitis displayed a normal-appearing mucosa with no inflammation. It has been suggested that patients with Candida esophagitis and intact immunity develop inflammation at the infected site that limits penetration by the microorganisms [26]. We have analyzed multiple clinico-epidemiologic parameters associated with systemic or local immunodeficiency, as well as some other known risk factors for Candida esophagitis, however the differences between cases with and without mucosal inflammation were not statistically significant.

Candida esophagitis may be caused by other Candida species, such as $C$. tropicalis, $C$. parapsilosis and $C$. glabrata; therefore we considered the possibility that $\mathrm{Can}$ dida esophagitis with mucosal inflammation and Candida esophagitis without inflammation could be caused by different Candida species [5, 6]. However, molecular analysis documented similarly high prevalence of $C$. albicans in both groups. The reason why some Candida infections do not induce mucosal inflammation remains unclear and warrants further investigation.

In conclusion, mucosal inflammation is present in the majority of Candida esophagitis cases commonly demonstrating epithelial regeneration, intraepithelial neutrophils and increased lymphocytes. Several histologic features may alert a pathologist contemplating the differential diagnosis of active esophagitis to the possibility of Candida etiology if fungal microorganisms are not appreciated. The concurrent presence of intraepithelial neutrophils and increased lymphocytes and, particularly, of superficial band-like neutrophils and peripapillary or CD4-predominant lymphocytes should trigger an active search for Candida microorganisms and for supportive endoscopic evidence of Candida esophagitis.

\section{Compliance with ethical standards}

Conflict of interest The authors declare that they have no conflict of interest.

\section{References}

1. Mathieson R, Dutta SK. Candida esophagitis. Dig Dis Sci. 1983;28:365-70.

2. Alsomali MI, Arnold MA, Frankel WL, et al. Challenges to "classic" esophageal candidiasis: looks are usually deceiving. Am J Clin Pathol. 2017;147:33-42.

3. Underwood JA, Williams JW, Keate RF. Clinical findings and risk factors for Candida esophagitis in outpatients. Dis Esophagus. 2003;16:66-9.

4. Haulk AA, Sugar AM. Candida esophagitis. Adv Intern Med. 1991;36:307-18.

5. Kliemann DA, Pasqualotto AC, Falavigna M, Giaretta T, Severo LC. Candida esophagitis: species distribution and risk factors for infection. Rev Inst Med Trop Sao Paulo. 2008;50:261-3.

6. Kakati B, Kotwal A, Biswas D, Sahu S. Fluconazole resistant Candida oesophagitis in immunocompetent patients: is empirical therapy justifiable? J Clin Diagn Res. 2015;9:DC16-18. 
7. Walsh TJ, Merz WG. Pathologic features in the human alimentary tract associated with invasiveness of Candida tropicalis. Am J Clin Pathol. 1986;85:498-502.

8. Moulinier B, Lambert R, Grenier-Boley P, Bruhiere J. Esophageal mycoses. Nouv Presse Med. 1972;1:2629-32.

9. Kodsi BE, Wickremesinghe C, Kozinn PJ, Iswara K, Goldberg PK. Candida esophagitis: a prospective study of 27 cases. Gastroenterology. 1976;71:715-9.

10. Demir D, Doganavsargil B, Sarsik B, Sezak M, Tuncyurek M. Is it possible to diagnose infectious oesophagitis without seeing the causative organism? A histopathological study. Turk J Gastroenterol. 2014;25:481-7.

11. Ismail-Beigi F, Horton PF, Pope CE 2nd. Histological consequences of gastroesophageal reflux in man. Gastroenterology. 1970;58:163-74.

12. Putra J, Muller KE, Hussain ZH, et al. Lymphocytic esophagitis in nonachalasia primary esophageal motility disorders: improved criteria, prevalence, strength of association, and natural history. Am J Surg Pathol. 2016;40:1679-85.

13. Munoz-Cadavid C, Rudd S, Zaki SR, et al. Improving molecular detection of fungal DNA in formalin-fixed paraffin-embedded tissues: comparison of five tissue DNA extraction methods using panfungal PCR. J Clin Microbiol. 2010;48:2147-53.

14. Martin R, Moran GP, Jacobsen ID, et al. The Candida albicansspecific gene EED1 encodes a key regulator of hyphal extension. PLoS ONE. 2011;6:e18394.

15. Xue Y, Suriawinata A, Liu X, et al. Lymphocytic esophagitis with CD4 T-cell-predominant intraepithelial lymphocytes and primary esophageal motility abnormalities: a potential novel clinicopathologic entity. Am J Surg Pathol. 2015;39:1558-67.

16. Geboes K, De Wolf-Peeters C, Rutgeerts P, et al. Lymphocytes and langerhans cells in the human oesophageal epithelium. Virchows Arch A Pathol Anat Histopathol. 1983;401:45-55.
17. Resnick MB, Finkelstein Y, Weissler A, Levy J, Yakirevich E. Assessment and diagnostic utility of the cytotoxic T-lymphocyte phenotype using the specific markers granzyme-B and TIA-1 in esophageal mucosal biopsies. Hum Pathol. 1999;30:397-402.

18. Lucendo AJ, Navarro $\mathrm{M}$, Comas $\mathrm{C}$, et al. Immunophenotypic characterization and quantification of the epithelial inflammatory infiltrate in eosinophilic esophagitis through stereology: an analysis of the cellular mechanisms of the disease and the immunologic capacity of the esophagus. Am J Surg Pathol. 2007;31:598-606.

19. Dunbar KB, Agoston AT, Odze RD, et al. Association of acute gastroesophageal reflux disease with esophageal histologic changes. JAMA. 2016;315:2104-12.

20. Conti HR, Shen F, Nayyar N, et al. Th17 cells and IL-17 receptor signaling are essential for mucosal host defense against oral candidiasis. JAMA. 2016;315:2104-12.

21. Hernandez-Santos N, Gaffen SL. Th17 cells in immunity to Candida albicans. Cell Host Microbe. 2012;11:425-35.

22. Muller KE, Putra J, Gabbard S, et al. Frequency and nature of increased intraepithelial lymphocytes in biopsies from patients with achalasia. Mod Pathol. 2014;27Suppl.2:194A.

23. Rubio CA, Sjodahl K, Lagergren J. Lymphocytic esophagitis: a histologic subset of chronic esophagitis. Am J Clin Pathol. 2006;125:432-7.

24. Sutton LM, Heintz DD, Patel AS, Weinberg AG. Lymphocytic esophagitis in children. Inflamm Bowel Dis. 2014;20:1324-8.

25. Daniels JA, Lederman HM, Maitra A, Montgomery EA. Gastrointestinal tract pathology in patients with common variable immunodeficiency (CVID): a clinicopathologic study and review. Am J Surg Pathol. 2007;31:1800-12.

26. Lauwers GY, Mino-Kenudson M, Kradin RL. Infections of the gastrointestinal tract. In: Kradin RL, editor. Diagnostic pathology of infectious disease. 1st ed. Elsevier, Philadelphia, PA; 2010. p. $215-54$. 